\title{
Odontogeriatria nos Projetos Políticos Pedagógicos dos cursos de Odontologia do sul do Brasil
}

\author{
Diogo Ogawa*; Maura Sassahara Higasi**; Pablo Guilherme Caldarelli**
}

* Estudante do Curso de Odontologia da Universidade
Estadual de Londrina
Docente do Departamento de Medicina Oral e
Odontologia Infantil, Universidade Estadual de
Londrina

\section{RESUMO}

O presente estudo quantitativo, exploratório e descritivo teve por objetivo apresentar um panorama situacional da inserção da Odontogeriatria nos Projetos Políticos Pedagógicos (PPP) dos cursos de Odontologia das instituições de ensino superior (IES) da Região Sul do Brasil. Os sítios Web dos cursos foram analisados, buscando-se as informações referentes à oferta da disciplina de Odontogeriatria nos PPP. Foram analisadas variáveis como natureza das instituições (pública ou privada), momento do curso no qual a disciplina é ofertada (ano/semestre), natureza do ensino (teórico, prático ou teórico-prático), carga horária total e disponibilidade de ementa, objetivos e conteúdos ministrados. Participaram do estudo 36 cursos de graduação em Odontologia das IES do sul do Brasil, dos quais 14 oferecem a disciplina de Odontogeriatria em sua matriz curricular, sendo seis (43\%) públicas e oito (57\%) privadas. Quanto à natureza da disciplina, em quatro cursos ela é teórica, em quatro é teórico/prática e seis cursos não disponibilizaram esta informação. Dez IES apresentaram ementa e conteúdos ministrados, sendo seis públicas e quatro privadas. O momento do curso no qual a disciplina é ofertada e a carga horária da mesma, apresentaram-se bastante heterogêneos. Não foram encontradas informações referentes aos PPP de três instituições, duas públicas e uma privada. $\mathrm{O}$ presente estudo possibilitou traçar um panorama da inserção da Odontogeriatria nas IES do sul do Brasil. Além disso, foi possível observar que nem todos os cursos de Odontologia disponibilizam informações sobre sua matriz curricular online, e quando estão disponíveis, nem todas as características são apresentadas.

Descritores: Educação em Odontologia. Ensino. Currículo. Odontologia Geriátrica.

\section{INTRODUÇÃO}

A população brasileira vem envelhecendo de maneira rápida. Sua estrutura etária, acompanhando o processo, sofre estreitamento progressivo da base da pirâmide populacional, com consequente aumento da expectativa de vida ${ }^{1,2}$. Desse modo, cada vez mais os cirurgiões-dentistas e demais profissionais da área da saúde têm encontrado números expressivos de pacientes idosos em seus ambientes de trabalho ${ }^{1,3}$.

Atualmente, os serviços odontológicos têm enfatizado a necessidade de que a saúde bucal seja concomitante à saúde geral, 
evitando que a primeira repercuta negativamente sobre a segunda ${ }^{3}$. Com relação à faixa etária populacional idosa, isso não é diferente. Entretanto, o cirurgião-dentista deve ter os conhecimentos necessários sobre a complexidade de um tratamento específico e diferenciado para o paciente idoso, abordando aspectos que envolvam suas condições sistêmicas, associadas ao processo normal do envelhecimento ${ }^{4}$.

Diante da inversão da pirâmide populacional brasileira, a Associação Brasileira de Ensino Odontológico (ABENO) empreendeu estudos que culminaram no desenvolvimento de uma proposta junto às diretrizes curriculares, a qual determina que temas relacionados à terceira idade devam integrar diferentes disciplinas que tratem de ciências sociais, de diagnóstico e planejamento de terapêuticas, de maneira que o estudante finalize seu curso atualizado em sua área profissional ${ }^{5}$.

Neste contexto, as Diretrizes Curriculares Nacionais $(\mathrm{DCN})^{6,7}$ para os cursos de Odontologia definem os objetivos do curso e os princípios na formação de cirurgiões-dentistas e se destacam como facilitadoras para a inserção destes conteúdos curriculares nos cursos de graduação em Odontologia. As DCN devem ter aplicação nacional e constituir o eixo orientador na elaboração dos currículos implementados por todas as instituições de ensino superior (IES), sendo complementadas por uma dimensão diversificada e específica, que reflita a experiência, a vocação de cada escola e as necessidades e imposições da região na qual se situa ${ }^{6-8}$.

Mudanças no ensino tradicional nas instituições acadêmicas, associadas ao cumprimento das DCN, têm sido realizadas visando diminuir o distanciamento entre a formação dos profissionais de saúde e as necessidades do Sistema Único de Saúde (SUS $)^{9}$. Dessa forma, com relação aos conteúdos de Odontogeriatria, torna-se importante proporcionar aos futuros cirurgiões-dentistas situações que permitam o desenvolvimento de habilidades e competências necessárias para o manejo adequado da população idosa, objetivando o atendimento universal, resolutivo, humanizado e de qualidade. Além disso, é de extrema importância que cirurgião-dentista conheça detalhadamente a biologia do envelhecimento e suas peculiaridades, bem como as inúmeras afecções e doenças bucais que compartilham fatores de risco das doenças sistêmicas para essa parcela etária ${ }^{3,4}$.

Com o intuito de cumprir os objetivos propostos pelas DCN, a apresentação dos Projetos Políticos Pedagógicos (PPP) das IES torna-se imprescindível para determinar os objetivos do curso, bem como os fundamentos, condições e procedimentos para a formação de cirurgiões-dentistas. Assim, objetivo do presente estudo foi apresentar um panorama situacional da inserção da Odontogeriatria nos PPP dos cursos de Odontologia da Região Sul do Brasil.

\section{MATERIAL E MÉTODO}

Trata-se de um estudo quantitativo, exploratório e descritivo, no qual o objeto do estudo compreendeu todos os cursos de Odontologia das IES da Região Sul do Brasil, segundo informações coletadas no sítio eletrônico do Conselho Federal de Odontologia $(\mathrm{CFO})^{10}$. Por se tratar de coleta de dados secundários, não houve necessidade de análise do projeto por comitê de ética em pesquisa.

Foram analisados todos os sítios destes cursos, buscando informações nos PPP sobre 
a inserção e oferta da disciplina de Odontogeriatria ou equivalentes nos currículos. Foram também analisadas as variáveis natureza das IES (pública ou privada), sua localização, o momento do curso no qual a disciplina é oferecida (ano/semestre), a natureza do ensino (teórico, prático ou teórico-prático), a carga horária total, a disponibilidade da ementa e os conteúdos ministrados.

Além disso, foram consultados documentos oficiais, bem como as legislações dos Ministérios da Educação e da Saúde relacionadas com a temática em questão.

\section{RESULTADOS}

No momento da coleta de dados foram encontradas 36 IES da Região Sul do Brasil que ofertam o curso de Odontologia, das quais 11 (31\%) são públicas e 25 (69\%) são privadas. Dessas IES, 14 apresentaram a disciplina de Odontogeriatria em seu PPP, sendo seis $(43 \%)$ instituições públicas e oito (57\%) privadas (Gráfico 1).

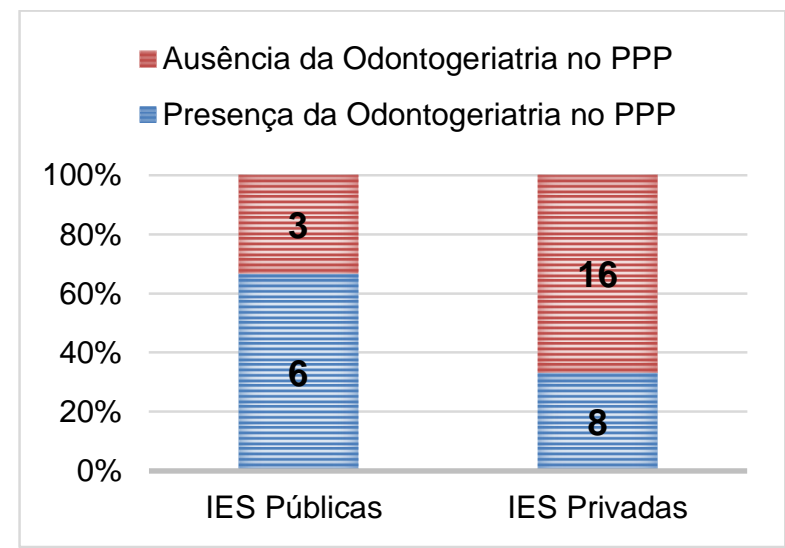

Gráfico 1 - Presença e ausência da disciplina de Odontogeriatria nos PPP dos cursos de Odontologia das IES públicas e privadas do sul do Brasil

$O$ restante das IES pesquisadas não apresentou em seus PPP a oferta da disciplina, totalizando 19 escolas, das quais três (16\%) são públicas e 16 (84\%) são privadas. Tornase importante ressaltar que, durante a coleta de dados do presente estudo, houve a impossibilidade de acesso ao PPP no sítio de três IES, duas públicas e de uma privada.

A distribuição geográfica das IES públicas e privadas do sul do Brasil que apresentam a disciplina de Odontogeriatria em seus PPP encontra-se esquematizada na Figura 1.

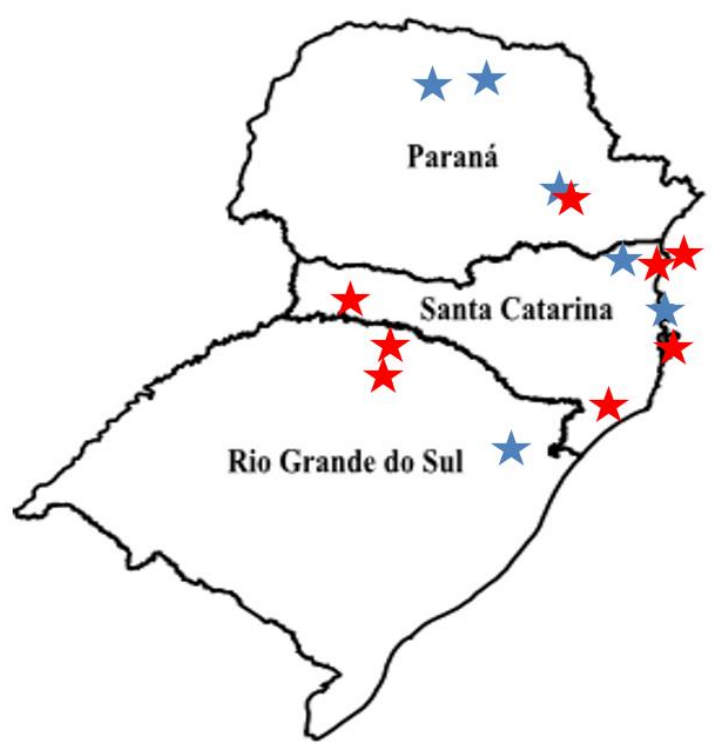

IES privadas $\star$ e públicas $\star$ com a disciplina de Odontogeriatria

Figura 1 - Distribuição geográfica das IES públicas e privadas do sul do Brasil que apresentam a disciplina de Odontogeriatria em seus PPP

O momento do curso no qual a disciplina é ofertada (ano/semestre), a natureza do ensino (teórico, prático ou teórico-prático) e carga horária total nas IES públicas e privadas, estão registrados nas tabelas 1 e 2 , respectivamente.

\section{DISCUSSÃO}

Com a tendência de aumento na expectativa de vida e o progresso da medicina 
Tabela 1 - Caracterização da disciplina de Odontogeriatria nos PPP das IES públicas da Região Sul do Brasil

\begin{tabular}{ccccc}
\hline Estado & $\begin{array}{c}\text { Número de } \\
\text { IES }\end{array}$ & Período & Ensino & $\begin{array}{c}\text { Carga horária } \\
\text { total (horas) }\end{array}$ \\
\hline PR & 1 & $4^{\circ}$ ano & Teórico & 10 \\
PR & 2 & $5^{\circ}$ ano & Teórico/Prático & 102 \\
SC & 1 & $7^{\circ}$ semestre & -- & 162 \\
SC & 1 & $10^{\circ}$ semestre & Teórico & 54 \\
RS & 1 & $8^{\circ}$ semestre & -- & -- \\
\hline
\end{tabular}

PR: Estado do Paraná

SC: Estado de Santa Catarina

RS: Estado do Rio Grande do Sul

- -: Dado não informado no Projeto Político Pedagógico.

Tabela 2 - Caracterização da disciplina de Odontogeriatria nos PPP das IES privadas da Região Sul do Brasil

\begin{tabular}{ccccc}
\hline Estado & $\begin{array}{c}\text { Número de } \\
\text { IES }\end{array}$ & Período & Ensino & $\begin{array}{c}\text { Carga horária } \\
\text { total (horas) }\end{array}$ \\
\hline PR & 1 & $9^{\circ}$ semestre & -- & 72 \\
SC & 1 & $6^{\circ}$ semestre & Teórico & 30 \\
SC & 2 & $6^{\circ}$ semestre & -- & $60 / 40$ \\
SC & 1 & $8^{\circ}$ semestre & Teórico/Prático & 60 \\
SC & 1 & $5^{\circ}$ Ano & -- & 72 \\
RS & 1 & $8^{\circ}$ semestre & Teórico & 36 \\
RS & 1 & $9^{\circ}$ semestre & Teórico/Prático & 60 \\
\hline
\end{tabular}

PR: Estado do Paraná

SC: Estado de Santa Catarina

RS: Estado do Rio Grande do Sul

- -: Dado não informado no Projeto Político Pedagógico.

e das demais áreas da saúde, têm sido gradualmente crescente a busca de cuidados por indivíduos com idade mais avançada. Tal fato salienta a importância de se traçar um panorama sobre o ensino da Odontogeriatria, que tem se tornado, cada vez mais, um importante campo de atuação para os cirurgiões-dentistas ${ }^{11}$.

Dessa forma, a inserção curricular da disciplina de Odontogeriatria nas IES pode ser traduzida como uma oportunidade gerada pelos cursos de Odontologia para que os acadêmicos entrem em contato com a abordagem e o tratamento odontológico do paciente idoso. No entanto, torna-se relevante destacar que a importância da disciplina de Odontogeriatria deve ser mais ampla do que simplesmente estar inserida nos currículos odontológicos ${ }^{12}$.

A literatura ${ }^{3,4,12}$ tem destacado que o estudante de Odontologia deve estar atento não apenas à apresentação de doenças relacionadas à saúde bucal nos idosos, como também ao grau de envolvimento dos fatores sociais e psicológicos relacionados a estas situações. Além disso, salienta que os 
acadêmicos devem estar aptos a distinguir as consequências normais e anormais do processo de envelhecimento, evitando estereotipar o paciente idoso i, $^{3,12}$.

A Região Sul do Brasil possui a segunda maior concentração de cursos de Odontologia do país ${ }^{13}$. Entretanto, os resultados encontrados no presente estudo demonstram que apenas $38,8 \%$ desses cursos apresentaram a disciplina de Odontogeriatria em seus PPP.

Dessa forma, considerando a demanda do atendimento odontológico ao idoso e os resultados encontrados, é possível observar que na região analisada, a faixa populacional em questão não se encontra completamente amparada, ratificando os resultados encontrados no estudo realizado por Nico $(2005)^{14}$, sobre a carência dos serviços odontológicos ao idoso.

O estudo realizado por Saintrain et al. $(2006)^{5}$ traçou o panorama do ensino da odontologia geriátrica nas Regiões Sul e Centro-Oeste do Brasil, mostrando que a disciplina se encontrava em fase de implantação nos cursos Odontologia. Este estudo também mostrou que, em 2006, nas regiões analisadas, dos 24 cursos de odontologia, apenas 18 responderam à pesquisa e sete apresentavam a disciplina de Odontogeriatria em seus currículos. Após cerca de dez anos, comparando os resultados encontrados no trabalho de Saintrain et al. $(2006)^{5}$ com os do presente estudo, é possível observar um expressivo aumento na implementação da disciplina nos currículos das IES, somando um total de 14 instituições com Odontogeriatria em seus PPP, entre públicas e privadas.

Outro aspecto relevante relacionado à oferta de disciplina de Odontogeriatria encontra-se vinculada à natureza das IES que as ofertam. Neste contexto, das 25 instituições privadas com o curso de odontologia no sul do país, apenas $32 \%$ oferecem a disciplina. Essa porcentagem se mostra muito inferior àquela encontrada nas IES públicas, que das onze sulbrasileiras com curso de odontologia, cerca de $55 \%$ apresentam a referida disciplina em seus currículos.

Tais dados devem ser melhor investigados, considerando formas alternativas de coleta de dados, como a utilização de questionários ou entrevistas com dirigentes das IES, aprimorando a compreensão desse panorama.

\section{CONCLUSÃO}

O presente estudo possibilitou traçar um panorama da inserção da Odontogeriatria nos PPP das IES do sul do Brasil, mostrando heterogeneidade com relação à natureza das IES, tipo de ensino, período de oferta e carga horária total da disciplina. Além disso, foi possível observar que nem todos os cursos de Odontologia disponibilizam informações sobre sua organização curricular online, e quando estão disponíveis, nem todas as características são apresentadas.

Dessa forma, os resultados encontrados reafirmam a necessidade da elaboração de novas pesquisas, utilizando outros métodos de coleta de informações, e a importância da transparência das IES em tornar públicos seus PPP.

\section{ABSTRACT \\ Geriatric Dentistry in Political Pedagogical Projects of Dentistry courses in Southern Brazil}

This is a quantitative, exploratory and descriptive study, which aimed to present a situational overview of the inclusion of geriatric dentistry in the Political Pedagogical Project (PPP) of Dentistry courses of Higher Education Institutions (HEI) from southern 
Brazil. The courses' websites were analyzed seeking information related to the offer of geriatric dentistry or equivalent discipline. It were also analyzed the nature of institutions (public or private), the State of the institutions, the period in which the course is offered (year/semester), nature of education (theoretical, practical or theoretical-practical), total workload, availability of menu, objectives and contents taught. From 36 graduation courses in dentistry analyzed, 14 offered geriatric dentistry discipline, six public and eight private. As for the nature of the discipline, in four courses it is theoretical, four are theoretical and practical and six courses didn't report this information. Ten HEIs presented menu and content taught, six public and four private. No information was found related to PPP of three institutions, two public and one private. This study made it possible to describe the present insertion of geriatric dentistry in PPP of HEI in southern Brazil. In addition, it was observed that not all dental courses provide information about their curriculum online, and when available, not all features are presented.

Descriptors: Education, Dental. Teaching. Curriculum. Geriatric Dentistry.

\section{REFRÊNCIAS}

1. Chaimowicz F. A saúde dos idosos brasileiros às vésperas do século XXI: Problemas, projeções e alternativas. Rev Saúde Públ. 1997;31(2):184-200.

2. Camarano AA. Os novos idosos brasileiros muito além dos 60 . Rio de Janeiro: IPEA; 2004.

3. Rosa AGF, Castellanos RA, Gomes-Pinto V. Saúde bucal na terceira idade. RGO. 1993;41(2):97-102.

4. Rosa LB, Zuccolotto MCC, Bataglion C, Coronatto EAS. Odontogeriatria - A saúde bucal na terceira idade. RFO. 2008;13(2):82-6.

5. Saintrain MVL, Souza EHA, Caldas Júnior AF. Ensino da Odontogeriatria nas faculdades de odontologia do sul e centro-oeste do Brasil: situação atual e perspectivas. Rev Odonto Ciênc. 2006; 21(53):270-7.

6. Brasil. CNE. Parecer CES/CNE 1300/2001 [internet]. Diário Oficial da União, Seção 1, p. 25; Brasília, 7 de dezembro de 2001 - [acesso em 20/09/2015]. Disponível em: http:// portal.mec.gov.br/cne/arquivos/pdf/CES 1300.pdf

7. Brasil. CNE. Resolução CNE/CES 3/2002 [internet]. Diário Oficial da União, Seção 1, p.10; Brasília, 4 de março de 2002 - [acesso em 20/09/2015]. Disponível em: http://portal.mec.gov.br/ cne/arquivos/pdf/CES032002.pdf

8. Carvalho ACP. Planejamento do curso de graduação de odontologia: É importante planejar os cursos de graduação considerando-se as Diretrizes Curriculares Nacionais. Rev ABENO. 2004; 4(1):7-13.

9. Carvalho BR, Costa TBC, Gomes MJ, Santos KT, Guerra SMG. Formação docente em odontologia no Brasil: sugestões de mudanças após as Diretrizes Curriculares Nacionais. Rev Bras Pesq Saúde. 2010;12(4):39-44

10. Conselho Federal de Odontologia - CFO [internet]. Consulta Faculdades de Odontologia por Estado - [acesso em: 20/09/2015]. Disponível em: http://cfo.org.br/servicos-e-consultas/ faculdades/

11. Brunetti R, Montenegro FLB. Odontogeriatria - noções de interesse clínico. São Paulo (SP): Editora Artes Médicas; 2002.

12. Newton JP. Health priorities and barriers to care of the older adult. Gerodontology. 2004;21(3):121-2. 
13. Paranhos LP, Ricci ID, Scanavini MA, Bérzin F, Ramos AL. Análise do mercado de trabalho odontológico na região Sul do Brasil. RFO. 2009;14(1): 7-13.

14. Nico LS. Formação de recursos humanos em odontologia quanto as disciplinas de gerontologia e Odontogeriatria [Dissertação]. São Paulo: Faculdade de Odontologia de Piracicaba (FOP) Universidade Estadual de Campinas (UNICAMP).
Correspondência para:

Pablo Guilherme Caldarelli e-mail: pablocaldarelli@ hotmail.com Rua Juiz de Fora, $n^{\circ}$ 230, Jardim Champagnat 86062-680, Londrina, PR 